\title{
Portrait: Fondation suisse pour l'éducation à la Santé
}

\author{
M. Schär et G. Bretscher, \\ Institut de Médecine Sociale et Préventive de \\ l'Université de Zurich
}

\begin{abstract}
Résumé 1
La Fondation suisse pour l'éducation de la santé a été fondée en 1972 sur l'initiative de la Société suisse de médecine sociale et préventive avec l'aide financière du Don suisse de la Fête nationale. Au conseil de fondation se trouvent représentées des institutions importantes (voir le texte allemand) qui sont intéressées à la promotion de l'éducation de la santé dans toute la Suisse. Les activités d'information, les prises de position officielles aussi bien que les conseils pour des organisations comptent parmi les tâches du "Centre de documentation de l'éducation pour la santé» à Zurich. Cette institution travaille en collaboration avec l'Institut de médecine sociale et préventive de l'Université de Zurich.

Pendant ses premières années d'existence, le Centre s'est surtout occupé de publier du matériel éducatif dans le domaine de la santé et de recueillir des productions audiovisuelles en vue du prêt. Son plus grand succès fut la publication des «Conseils médiaux pour les voyages dans les pays tropicaux», dont la première édition a paru en 1975 en allemand et en français. Mais les limites financières et en personnel dans la réalisation de tels projets sont vite devenues des évidences.
\end{abstract}

\footnotetext{
${ }^{1}$ Le texte intégral en langue allemande paraîtra dans le no. 6/1977 de cette Revue.
}

Entre temps, les tâches du Centre ont été modifiées, en tenant compte des changements de notre société et de son économie: comme l'intérêt commun aux problèmes d'épidémiologie et de médecine préventive a augmenté au fur et à mesure que les dépenses dans le secteur sanitaire croissaient, on a estimé qu'une intense éducation pour la santé fait en tout cas partie de la solution de ces problèmes. En réalité, compte tenu de la complexité du problème, il existe une carence nette en matière de coordination, de coopération, des moyens disponibles aussi que des idées et propositions.

Pour cette raison, le Centre s'occupe aujourd'hui surtout des tâches qui seront indispensables pour l'éducation pour la santé à l'avenir:

1. Coordination: Constitution d'un «inventaire pédagogique sanitaire».

2. Coopération et conseils: Etablissement de contacts entre des institutions isolées, informations sur les institutions, matériels et activités.

3. Recherche: Participation aux programmes de recherche pour l'évaluation de l'efficacité des stratégies pédagogiques, sanitaires; développement des techniques d'évaluation.

4. Promotion de la philosophie de l'éducation pour la santé: Publicité et activités de pointe pour progager et promouvoir la pédagogie sanitaire. 